\title{
Hybrid Surgical and Radiological Revascularization for Diabetic and Non-Diabetic Lower Limb Ischaemia
}

\author{
Harun Thomas ${ }^{\mathrm{a}}$, Mohamed Aly ${ }^{\mathrm{a}}$, Mei Teng Wong ${ }^{\mathrm{a}}$, Daniel Moffat ${ }^{\mathrm{a}}$, Simon Mackenzie ${ }^{\mathrm{a}}$, \\ Sohail Choksy ${ }^{\mathrm{a}}$, Christopher Backhouse ${ }^{\mathrm{a}}$, Mohammad Al-Dabbaga ${ }^{\mathrm{a}}$, Adam Howard ${ }^{\mathrm{a}, \mathrm{b}}$
}

\begin{abstract}
Background: Multi-level occlusive arterial disease involving the iliac and distal arteries can be treated with major bypass, sequential or single stage radiological and surgical procedures. We report our experience with the single stage hybrid approach.
\end{abstract}

Methods: All patients who underwent hybrid intervention between June 2008 and June 2011 were reported. Patients deemed medically unfit for major bypass or with insufficient veins were mainly considered. Prospective database of patients, procedures and complications with retrospective review of inpatient, outpatient and operative notes, computed tomographic angiography and duplex scan was performed.

Results: Twenty three patients underwent 24 single stage procedures. The patient age ranged from 49 to 88 years (mean 70 ) with 19 males. Fifteen $(65 \%)$ patients presented with intermittent claudication, $12(52 \%)$ with short distance $(\leq 100$ yards) claudication, $12(52 \%)$ with tissue loss and $8(35 \%)$ with rest pain. Overall, 18 (78\%) patients were presented with critical ischemia limb. The commonest procedures were iliac angioplasties with endarterectomies or bypasses, 23 out of 24 procedures (96\%) were technically successful and 20 out of 22 patients (91\%) followed up had clinically successful outcome. The mean ankle brachial pressure index improved from 0.57 to 0.71 . Two patients ( $9 \%$ ) died, 2 patients (9\%) developed severe complications and 6 patients (26\%) developed moderate complications within 30 days.

Conclusions: Single stage hybrid radiological and surgical procedure for lower limb ischemia is feasible providing high technical and clinical success in patients unfit for major bypass or with limited vein conduit. It saves one admission providing patient conve-

\footnotetext{
Manuscript accepted for publication August 15, 2012

anstitution- Colchester General Hospital, Turner Road, Colchester, Essex, CO4 5JL, United Kingdom

${ }^{\mathrm{b} C o r r e s p o n d i n g ~ a u t h o r: ~ A d a m ~ H o w a r d, ~ C o n s u l t a n t ~ S u r g e o n, ~}$

Colchester General Hospital, Turner Road, Colchester, Essex, CO4 5JL,

United Kingdom. Email: adam.howard@colchesterhospital.nhs.uk

doi: http://dx.doi.org/10.4021/jcs101w
}

nience and potential cost savings.

Keywords: Lower limb ischemia; Hybrid procedure; Single stage

\section{Introduction}

Multi-level atherosclerotic arterial occlusion is the commonest presentation of symptomatic peripheral vascular disease (PVD) in the western population [1]. Intervention to correct multilevel PVD has traditionally been performed as a staged procedure: usually a radiological intervention to establish the inflow and a surgical procedure to enable adequate runoff. There have only been few reports of combined 'single stage' radiological and surgical intervention for correction of multi-level arterial occlusive disease. A single stage hybrid procedure offers potential benefits like avoiding a hospital admission for the pre-surgical radiological procedure; easier radiological access in a completely occluded artery; feasible in patients unfit for major bypass; possible even if there is inadequate vein for major bypass and provides immediate distal runoff. However this may be at the expense of increase recovery time and complications after a longer theatre procedure and general anaesthetic. We report our experience with single stage approach looking at the patient presentation, procedures performed, results, complications and follow-up.

\section{Materials and Methods}

The details of patients were obtained from a prospectively maintained database of all patients undergoing hybrid single stage radiological and surgical procedure at our hospital. Retrospective reviews of inpatient notes, operative notes, computed tomographic angiography (CTA), duplex scan reports, and outpatient clinic notes were performed. The inclusion criteria were patients presenting with peripheral occlusive vascular disease who had hybrid single stage surgical and radiological procedures between June 2008 and June 2011. All patients had preoperative CTA. Presence of significant arterial disease was defined by stenosis more than $50 \%$ on 
Table 1. Combined Single Stage Surgical and Radiological Intervention for Lower Limb Ischemia

\begin{tabular}{ll}
\hline Study Period & June 2008 to June 2011 \\
Number of Procedures & 24 procedures in 23 patients \\
Technical Success & 23 out of 24 procedures $(96 \%)$ \\
Clinical Success & 20 out of 22 patients followed up $(91 \%)$ \\
Major amputation free survival & 22 out of 23 patients followed up $(97 \%)$ \\
30 day readmission & $1(4.5 \%)$ \\
30 day Mortality & $2(9 \%)$ \\
30 day major complication & $2(9 \%)$ \\
\hline
\end{tabular}

CT angiography or critical ischaemia. Patients selected for the hybrid procedure were those who had significant PVD in the iliac or common femoral arteries, treatable distal disease, inadequate vein for bypass, and poor medical candidates that were high risk for major bypass surgery. Intraoperative angiography was performed to confirm these findings.

All procedures were performed by the surgeon and the radiologist with patient under general anaesthesia in the operating theatre. Femoral arterial exposure and control was gained in the standard manner. Seldinger technique with fluoroscopic guidance was used to obtain retrograde guide wire access. Standard infra-inguinal surgical procedures were then performed with the guide wire in place. A retrograde radiological sheath was then placed through the anastomosis suture line, patch incision or vein conduit side branch tributary. Standard interventional techniques were then used to treat the proximal or distal arterial lesion. If initial guide wire access was difficult, it was then attempted after arteriotomy or endarterectomy. Contralateral approach was never required for guide wire placement.

The data collected included, demographics (age and sex), vascular risk factor co morbidities (diabetes, IHD, smoking status, hypertension, cerebrovascular accidents, and hypercholesterolemia), presenting symptoms (claudication pain, rest pain, ulceration or gangrene), pre-operative investigations (CTA, Duplex), type and site of surgical and radiological procedures, diameters of balloons, types of stents, length of stay, length of recovery, technical, clinical, and haemodynamic success, postoperative complications, preoperative and postoperative ankle brachial pressure index (ABPI), and follow up.

Technical success was defined as successful technical execution, good post-angioplasty angiographic appearance, the absence of immediate intra-operative complications, good pulses palpable distal to the occlusion and improved distal perfusion. Clinical success was defined as improved post-operative ankle-brachial pressure index and according to the Rutherford categories: considered successful if they improved by one category and considered as clinical failure if they showed no improvement or deteriorated. Haemodynamic success was defined as postoperative duplex or CT angiography showing patent stent/bypass and biphasic good flow in the operated segment compared to preoperative investigations. Any complications within 30 days of surgery were recorded. Peri-operative mortality was described as death within 30 days of the procedure. Data was analyzed and expressed as total, range, mean and median as applicable.

\section{Results}

Between June 2008 and June 2011, 23 symptomatic patients underwent 24 elective or emergency hybrid surgical and radiological interventions for lower limb ischaemia. The patient age ranged from 49 to 88 (mean 70) with 19 males. Almost all were smokers or ex-smokers (96\%), 11 (48\%) had history of hypertension, 11 (48\%) were diabetics, 8 (35\%) had a history of ischaemic heart disease, $8(35 \%)$ had history of hypercholesterolemia, and $5(22 \%)$ had history of cerebrovascular disease, $15(65 \%)$ patients presented with intermittent claudication pain; $12(52 \%)$ of whom presented with short distance ( $\leq 100$ yards) claudication, $12(52 \%)$ presented with ischaemic ulcers or gangrene and $8(35 \%)$ had rest pain. Overall, 18 out of $23(78 \%)$ patients presented with critical ischemia limb. (persistent ulcers, night/rest pain or no signals on Doppler scan) (Table 1).

The radiological procedures performed were 14 common iliac artery angioplasties (58\%) and 11 external iliac artery angioplasties (46\%) and one popliteal stent. The mean balloon diameter used was $8 \mathrm{~mm}(6 \mathrm{~mm}-10 \mathrm{~mm}), 7$ stents were put in 5 patients ( 5 optimed supaflex self-expanding ntinol, 1 sinus supaflex self expanding, 1 viabahn stent).

The surgical interventions performed were $15(63 \%)$ 
common femoral (CFA) endarterectomies, 7 (29\%) femoropopliteal bypass, $4(17 \%)$ profundoplasties, and $4(17 \%)$ femoro-femoral crossovers,. 3 (13\%) operations combined both CFA endarterectomies and femoro-popliteal bypasses and $2(8 \%)$ operations combined both CFA endarterectomies with femoro-femoral crossovers, $2(8 \%)$ procedures used hemashield patches and $2(8 \%)$ used long saphenous vein patch. One operation included repairing 3 aneurysms along the PFA, and popliteal artery.

The median post-operative hospital stay was 6 days ( 3 46 days). The mean follow-up was 208 days (23 - 707 days), 23 out of 24 procedures $(96 \%)$ were technically successful, while in 1 procedure angioplasty of the tibioperoneal trunk was abandoned due to poor distal run-off. Mean pre-op ABPIs were 0.57 (unrecordable - 0.84), while post-op ABPIs averaged at $0.71(0.35-1.08), 12$ patients had follow-up duplex scans or CTA, with haemodynamic success recorded in 9 , and haemodynamic failure recorded in 3 patients due to damped distal flow, or significant stenosis post-operatively.

Twenty-two out of the 23 patients had follow-up data available. The median Rutherford category pre-operatively was 4 (range 2 - 6). Clinical success was recorded in 20 (91\%) patients: 13 reached complete resolution of symptoms (cat. 0 ) and 7 patients showed improvement in their symptoms. Clinical failure occurred in 2 patients; one patient developed a groin infection and a critically ischemic limb that required re-admission while the other patient developed wet gangrene of the great toe that required amputation prior to discharge and subsequently required further revascularisation. Median post-operative Rutherford category was 0 (range 0 - 5). None of the 22 patients followed-up required major amputation giving a $97 \%$ amputation free survival.

Post operative complications were classified according to Accordion Severity Grading of Postoperative Complications [2]: 2 patients developed severe complications (wound infection requiring exploration, and wet gangrene of the great toe requiring amputation) and 6 complications were categorized as moderate (4 surgical site infections and one urinary tract infection which all resolved on antibiotics and 1 patient with post-operative pyrexia and tachycardia). There were $2(9 \%)$ post-operative deaths within 30 days due to bowel ischemia that required a laparotomy from which the patient didn't recover, and the other patient suffered from excessive bleeding and though transfused suffered from acute on chronic renal failure and passed away in ITU.

\section{Discussion}

Treatment of multi-level occlusive disease involving the iliac arteries and distal vessels has traditionally been performed by staged radiological and surgical intervention. In patients with occlusive disease of the external iliac artery extending to the common femoral artery, pure endovascular approach is usually avoided because stenting across the inguinal ligament results in significantly poorer outcomes due to damage of stents across a moving joint and an increased development of intimal hyperplasia at these locations [3, 4]. The hybrid procedure allows the endarterectomy to just above the inguinal ligament with angioplasty and stent to the proximal common/external iliac segments. The hybrid single stage approach may provide potential advantages: improved access to the arteries especially in cases of complete occlusion, shorter length of vein required, single admission with potential cost advantages and patient convenience, smaller surgical procedures and scars.

One of the first reports of combined procedure was by Brewster et al [5] who, after combined iliac angioplasty and distal reconstruction, achieved a $76 \% 5$-year patency rate that improved to an $88 \%$ assisted patency rate with reintervention. Further reports [6-10] have confirmed the good long term results of combined procedure. Nelson et al [11] has stressed the importance of placing the guide wire for angioplasty before performing the endarterectomy to reduce the chance of dissection and for technical ease. In one of the largest series so far Chang et al [12] reported on 171 patients who underwent 193 CFA endarterectomises and iliac stent/ stent grafting with a technical success of $98 \%$ of patients and long term clinical improvement in $92 \%$ of patients. The mean ankle-brachial index increased from 0.38 to 0.72 with a thirty-day mortality of $2.3 \%$ and a 5 -year survival of $60 \%$. Our results show a $96 \%$ (23 out of 24) immediate technical success with a $91 \%$ (20 out of 22) clinical success and an improvement of mean ABPI from 0.57 to 0.72 after the procedure.

Primary endovascular procedure is often difficult in the presence of a completely occluded femoral artery. In the hybrid procedure, an initial femoral endarterectomy can provide access for the radiological intervention. Furthermore, the hybrid procedure provides an immediate outflow that may increase inflow patency in comparison to the staged procedure where the outflow post-angioplasty/stent is often diseased. Single stage hybrid procedure could avoid two inpatient episodes which will be more convenient for the patients. This could potentially reduce costs thought we have not formally compared the cost differences. The median length of postoperative stay was 6 days which compares favourably with patients who undergo bypass surgery for infra-inguinal peripheral vascular disease [13, 14].

Recently more vascular surgeons are developing endovascular skills with favourable results $[11,15,16]$. In the United Kingdom, recent proposals recommend the incorporation of endovascular skills into vascular surgical training. This would increase the applicability of single stage hybrid radiological and surgical procedures however we recommend a radiologist and surgeon should work in tandem. The limitations of this study are its retrospective nature, limited numbers and lack of comparison with the traditional surgical 
approach.

Single stage hybrid radiological and surgical procedure for multilevel lower limb peripheral vascular disease is feasible providing high technical, hemodynamic and clinical success. It saves one admission with associated patient convenience and potential cost savings. Further comparative studies are required to assess the complications and cost savings.

\section{Conflict of Interest}

All authors declare that there are no conflicting or competing financial interests to declare regarding this article.

\section{Grant Support}

No grants obtained.

\section{Disclosures}

No financial disclosures.

\section{References}

1. Mousa A, Abdel-Hamid M, Ewida A, Saad M, Sahrabi A. Combined percutaneous endovascular iliac angioplasty and infrainguinal surgical revascularization for chronic lower extremity ischemia: preliminary result. Vascular. 2010;18(2):71-76.

2. Strasberg SM, Linehan DC, Hawkins WG. The accordion severity grading system of surgical complications. Ann Surg. 2009;250(2):177-186.

3. Andrews RT, Venbrux AC, Magee CA, Bova DA. Placement of a flexible endovascular stent across the femoral joint: an in vivo study in the swine model. J Vasc Interv Radiol. 1999;10(9):1219-1228.

4. Ballard JL, Sparks SR, Taylor FC, Bergan JJ, Smith DC, Bunt TJ, Killeen JD. Complications of iliac artery stent deployment. J Vasc Surg. 1996;24(4):545-553; discussion 553-545.

5. Brewster DC, Cambria RP, Darling RC, Athanasoulis CA, Waltman AC, Geller SC, Moncure AC, et al. Long-term results of combined iliac balloon angioplasty and distal surgical revascularization. Ann Surg. 1989;210(3):324-330; discussion 331.

6. Aburahma AF, Robinson PA, Cook CC, Hopkins ES.
Selecting patients for combined femorofemoral bypass grafting and iliac balloon angioplasty and stenting for bilateral iliac disease. J Vasc Surg. 2001;33(2 Suppl):S93-99.

7. Demasi RJ, Snyder SO, Wheeler JR, Gregory RT, Gayle RG, Parent FN, Gandhi RH. Intraoperative iliac artery stents: combination with infra-inguinal revascularization procedures. Am Surg. 1994;60(11):854-859.

8. Faries PL, Brophy D, LoGerfo FW, Akbari CM, Campbell DR, Spence LD, Hook SC, et al. Combined iliac angioplasty and infrainguinal revascularization surgery are effective in diabetic patients with multilevel arterial disease. Ann Vasc Surg. 2001;15(1):67-72.

9. Perler BA, Williams GM. Does donor iliac artery percutaneous transluminal angioplasty or stent placement influence the results of femorofemoral bypass? Analysis of 70 consecutive cases with long-term follow-up. J Vasc Surg. 1996;24(3):363-369; discussion 369-370.

10. Marin ML, Veith FJ, Sanchez LA, Cynamon J, Suggs WD, Schwartz ML, Parsons RE, et al. Endovascular aortoiliac grafts in combination with standard infrainguinal arterial bypasses in the management of limbthreatening ischemia: preliminary report. J Vasc Surg. 1995;22(3):316-324; discussion 324-315.

11. Nelson PR, Powell RJ, Schermerhorn ML, Fillinger MF, Zwolak RM, Walsh DB, Cronenwett JL. Early results of external iliac artery stenting combined with common femoral artery endarterectomy. J Vasc Surg. 2002;35(6):1107-1113.

12. Chang RW, Goodney PP, Baek JH, Nolan BW, Rzucidlo EM, Powell RJ. Long-term results of combined common femoral endarterectomy and iliac stenting/stent grafting for occlusive disease. J Vasc Surg. 2008;48(2):362-367.

13. Fowkes F, Leng GC. Bypass surgery for chronic lower limb ischaemia. Cochrane Database Syst Rev. 20082):CD002000.

14. Ah Chong AK, Tan CB, Wong MW, Cheng FS. Bypass surgery or percutaneous transluminal angioplasty to treat critical lower limb ischaemia due to infrainguinal arterial occlusive disease? Hong Kong Med J. 2009;15(4):249-254.

15. Hamilton IN, Jr., Mathews JA, Sailors DM, Woody JD, Burns RP. Combination endovascular and open treatment of peripheral arterial occlusive disease performed by surgeons. Am Surg. 1998;64(6):581-590; discussion 590-582.

16. Gross GM, Johnson RC, Roberts RM. Results of peripheral endovascular procedures in the operating room. $\mathrm{J}$ Vasc Surg. 1996;24(3):353-361; discussion 361-352. 